\title{
La Chine au risque de la dépendance alimentaire
}

\author{
Jean-Marc CHAUMET ${ }^{1}$ \\ Thierry $\mathrm{POUCH}^{2}$ \\ 1 Département économie de I'Institut de \\ I'élevage, \\ 149 Rue de Bercy, \\ 75595 Paris Cedex 12 \\ $<$ jean-marc.chaumet@idele.fr > \\ 2 Direction économie des agricultures et \\ des territoires de I'APCA, \\ 9, Avenue George V, \\ 75008 Paris et Laboratoire REGARDS, \\ Université de Reims Champagne Ardenne, \\ 57 rue Pierre Taittinger, \\ 51096 Reims Cedex
}

Article reçu le 18 juillet 2012

Accepté le 30 juillet 2012

L'économie chinoise suscite autant d'inquiétudes, d'admiration que d'espoirs. Inquiétudes quant à la fulgurance de sa progression économique, faisant dire qu'elle prend une part active au basculement du centre de gravité qui est en train de se produire dans l'économie mondiale. Admiration dans le sens où sa capacité de renouvellement des structures productives a été des plus rapides et efficaces depuis la fin des années soixante-dix. L'insertion de la Chine dans la division internationale du travail l'a propulsée premier exportateur mondial de marchandises en 2010, surclassant I'Allemagne. À suivre l'évolution de la Chine depuis 1978, I'objectif demeure le même : rattraper puis dépasser I'Occident de façon à élever le niveau de bienêtre de la population, qui représente $25 \%$ de la population mondiale. Espoirs aussi car, dans la crise actuelle, le redéploiement de la croissance vers le marché intérieur chinois pourrait tirer de façon décisive la croissance mondiale.

\begin{abstract}
China close to the point of food dependency
China's opening to the world and economic reforms began in 1978 and resulted in remarkable economic growth. Food transition then occurred for part of Chinese citizens, meaning more meat consumption and less grain consumption. Many factors explained this transition. As China's economy grew rapidly, peoples' average income have been rising and cities have been growing at a rapid pace. Foreign investments in retail also have played a major role. But some gaps remain throughout the country: income inequality between rural and urban, between people living on the coast and in-land people, among generations. . Food transition is not over yet for an important part of the Chinese population.

China was able to feed its population until the beginning of the 21 st century. But the imports of soybean and milk products have widened its food trade deficit since 2004. The crisis that has been affecting the global economy for the past 4 years might force the Chinese authorities to boost home consumption to be able to maintain the economic growth. This move would mean more imports and might have big consequences on food international trade in the years to come.
\end{abstract}

Key words: China, economic growth, food transition, food consumption, food trade, soybean import

Ces perspectives interpellent : comment la Chine va pouvoir à la fois rattraper I'Occident, se hisser au rang de puissance hégémonique dans le secteur industriel et nourrir une population qui, en 2030, devrait avoisiner 1,4 à 1,5 milliard d'individus ? Car la dynamique de la croissance en Chine a évidemment produit de très profondes mutations structurelles, économiques et sociales. Parmi elles figure l'alimentation, pour laquelle la Chine est exposée à une double contrainte: maîtriser sur une longue période l'approvisionnement quantitatif en biens alimentaires de sa population, et satisfaire des besoins en transformation radicale. Quelles sont les répercussions sur les importations chinoises de produits agricoles et alimentaires?

La première partie de l'article traitera de l'ouverture de la Chine aux modes de vie occidentaux, et de sa conséquence première : elle est devenue progressivement un importateur de premier ordre de biens alimentaires. La deuxième reviendra sur les traits saillants de la mutation de la consommation alimentaire. Enfin, la dernière partie montrera en quoi la Chine est exposée au risque de ne plus pouvoir, à plus ou moins long terme, nourrir sa population, l'obligeant à recourir chaque jour davantage aux importations. Les signes annonciateurs d'un tel scénario sont illustrés par l'évolution même des échanges de produits agricoles et alimentaires de la Chine avec le reste du monde.

\section{Croissance chinoise et mutations économiques et sociales}

Le gouvernement chinois a récemment revu à la baisse ses prévisions de croissance économique. Affectée par le ralentissement de l'économie mondiale et par la crise de la Zone euro, la croissance du PIB a enregistré quelques

Pour citer cet article : Chaumet JM, Pouch T. La Chine au risque de la dépendance alimentaire. OCL 2012; 19(5) : 290-298. doi : 10.1684/ ocl.2012.0466 
revers depuis le déclenchement de la crise en août 2007. La Chine est I'un des pays les plus exposés au commerce mondial de marchandises: les flux totaux de marchandises de cette économie représentant près de $65 \%$ du PIB. Les effets négatifs de la crise sur les échanges ont ainsi joué un rôle crucial de frein à la croissance (modèle dit export led growth). II n'en demeure pas moins que la croissance se situe toujours sur un rythme élevé, plus de $9 \%$ en 2011, chiffre attendu dans une fourchette de 7-7,5\% en 2012 (figure 1).

Depuis le début de la décennie quatrevingt-dix, le taux de croissance du PIB n'est plus jamais descendu en deçà de $8 \%$. C'est d'ailleurs à partir de cette époque que la Chine s'est transformée en une sorte de pivot du système industriel mondial. On a pu en déduire que la Chine était l'un des pays émergents les plus résistants à la crise (Lemoine, 2009). Une telle résistance est pour partie liée à la réactivité des autorités chinoises : elles ont injecté en 2010 près de 600 milliards de dollars dans l'économie afin d'atténuer les effets de la crise (soit un plan de relance de l'ordre de $15 \%$ du PIB) et la Banque Centrale a réduit ses taux d'intérêt.

Mais le modèle de croissance chinois repose toujours essentiellement sur les exportations. Les menaces que font peser la crise sur les exportations chinoises conduisent les économistes à escompter un recentrage de la croissance sur la demande intérieure, c'est-à-dire sur la consommation des ménages. II s'agit d'une perspective de moyen voire de long terme, en raison des nombreuses variables macroéconomiques qu'il va falloir faire évoluer (politique salariale, épargne, protection sociale...) (Apoteker, 2009).

L'ampleur de la croissance en Chine depuis la fin des années soixante-dix a occasionné deux types de mutations structurelles. La première a été la formation d'une véritable classe moyenne. Selon les données issues de Chinese Household Income Project (CHIP), la pauvreté en Chine a fortement diminué entre 1995 et 2007, la part de la population disposant d'un revenu journalier compris entre 6 et $10 \$$ passant de $4,8 \%$ à $25,5 \%$, celle se situant dans la tranche supérieure (10-20 \$ par jour) étant passée de 0,7 à 18,7\%. Cette progression de la classe moyenne chinoise ne concerne pas uniquement que

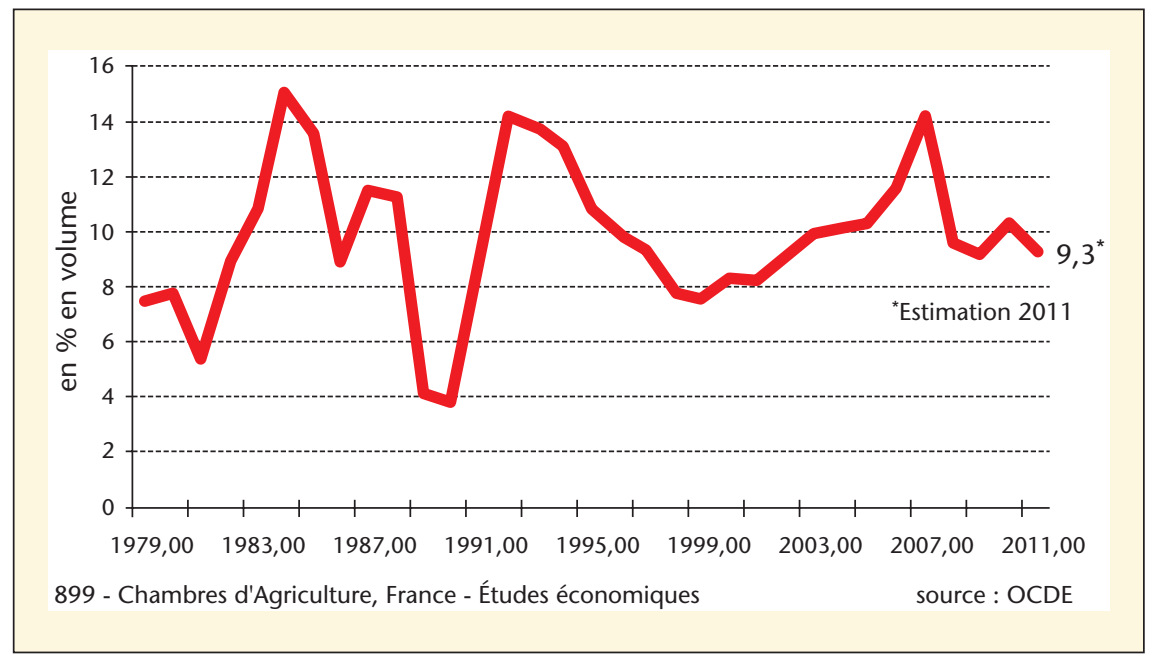

Figure 1. Taux de croissance du PIB de la Chine (en \% en volume).

les régions urbaines. On constate en effet une progression de celle-ci y compris dans les zones rurales ${ }^{1}$. La seconde concerne le processus d'urbanisation qui est à l'œuvre depuis les années quatre-vingt. Les chiffres des Nations Unies indiquent que d'à peine $20 \%$ en 1980 , la population urbaine a bondi à $26 \%$ en 1990 et à $50 \%$ en 2010 . Cette tendance à l'urbanisation de la population devrait se confirmer à l'horizon 2030, puisque ce ne seront pas moins de $70 \%$ des Chinois qui vivront en ville selon les projections de $\mathrm{I}^{\prime} \mathrm{ONU}^{2}$. Cette accélération de l'urbanisation de la population chinoise est à rapprocher de la dynamique d'industrialisation qui caractérise la Chine depuis près de quarante ans, dont les répercussions sur les disponibilités en terres agricoles constituent une source de préoccupation. C'est ce quadriptique " démographiecroissance-niveau de vie-industrialisation " qui avait fait dire à Lester Brown, en 1994, que la Chine sera de plus en plus dépendante de l'extérieur pour son approvisionnement alimentaire (Brown, 1995) (He et Simon, 2005).

Les implications de la formation d'une classe moyenne et de l'urbanisation sont nombreuses. Parmi elles figure la croissance de la demande de biens de consommation durables et non durables. Grâce à un revenu qui ne cesse de

${ }^{1}$ Cf. Key Indicators for Asia and Pacific, 2010, Banque asiatique de développement (www. adb.org)

${ }^{2}$ http://esa.un.org/unpd/wup/Country-Profiles/country-profiles_1.htm progresser, les ménages chinois ont augmenté leurs dépenses de consommation, et se sont équipés en appareils électroniques grand public, en électroménager, en automobiles et autres matériels de transport comme les scooters et motos. . II faut y voir une tendance à l'occidentalisation des comportements de consommation, tendance qui touche manifestement également la consommation alimentaire. L'un des symboles de cette ouverture aux produits alimentaires occidentaux est l'explosion récente des importations chinoises de vins et boissons alcoolisées, notamment en provenance de France (figure 2).

\section{Les transformations des modes de consommation alimentaire depuis 1978}

Cette croissance économique continue a donc impulsé l'évolution des consommations alimentaires en Chine, comme le montrent les données publiées par les autorités chinoises et la FAO ${ }^{3}$. Les statistiques chinoises, bien qu'incomplètes et d'une fiabilité relative (Ma et al.,

\footnotetext{
${ }^{3}$ Les données de la FAO sont issues du site FAOSTAT. Par souci de simplification, nous assimilerons les disponibilités alimentaires aux consommations alimentaires tout en gardant à l'esprit qu'il ne s'agit que d'une approximation : les disponibilités mesurent la quantité de calories à disposition des consommateurs et incluent donc les pertes qui peuvent survenir entre l'achat et l'ingestion.
} 


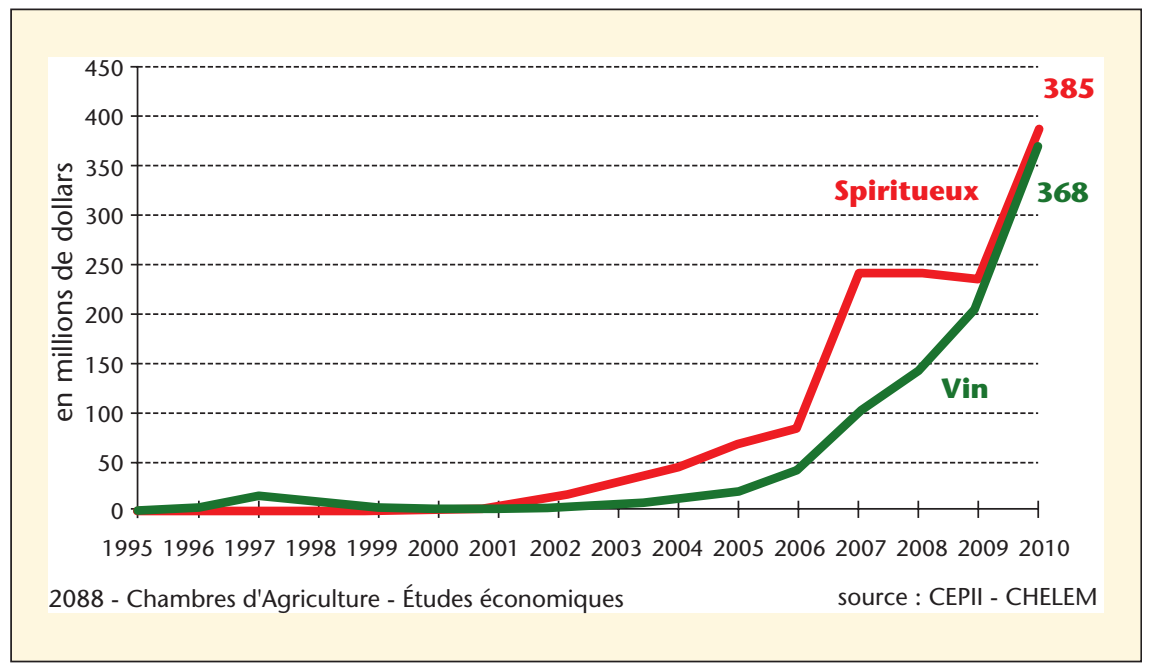

Figure 2. Exportations françaises de vins et spiritueux vers la Chine en millions de dollars.

2004) et même parfois contradictoires (Aubert, 2008) permettent cependant d'appréhender les grandes évolutions de la consommation à l'échelle du pays. Les tendances observées à travers l'étude de ces données sont par ailleurs corroborées par des études de terrain réalisées par de nombreux chercheurs (Du et al., 2004) (Fu et al., 2006) (Zhai et al., 2009).

\section{Transition du végétal à l'animal}

Le régime traditionnel chinois était composé d'une part importante de féculents, associés en moindres quantités à des légumes et des produits animaux (Sabban, 2010). La ration alimentaire était pauvre en lipides. Ce régime s'est considérablement modifié au cours des 50 dernières années. II a globalement suivi, avec du retard, les évolutions connues en Europe et en France (Combris et Soler, 2011). La première étape débute au lendemain du "Grand bond en avant", à l'orée des années 1960. À cette époque, les disponibilités annuelles par habitant étaient de moins de 1500 kcal, quasi exclusivement constituées de végétaux (96\%). En grande majorité, il s'agissait de céréales (riz et

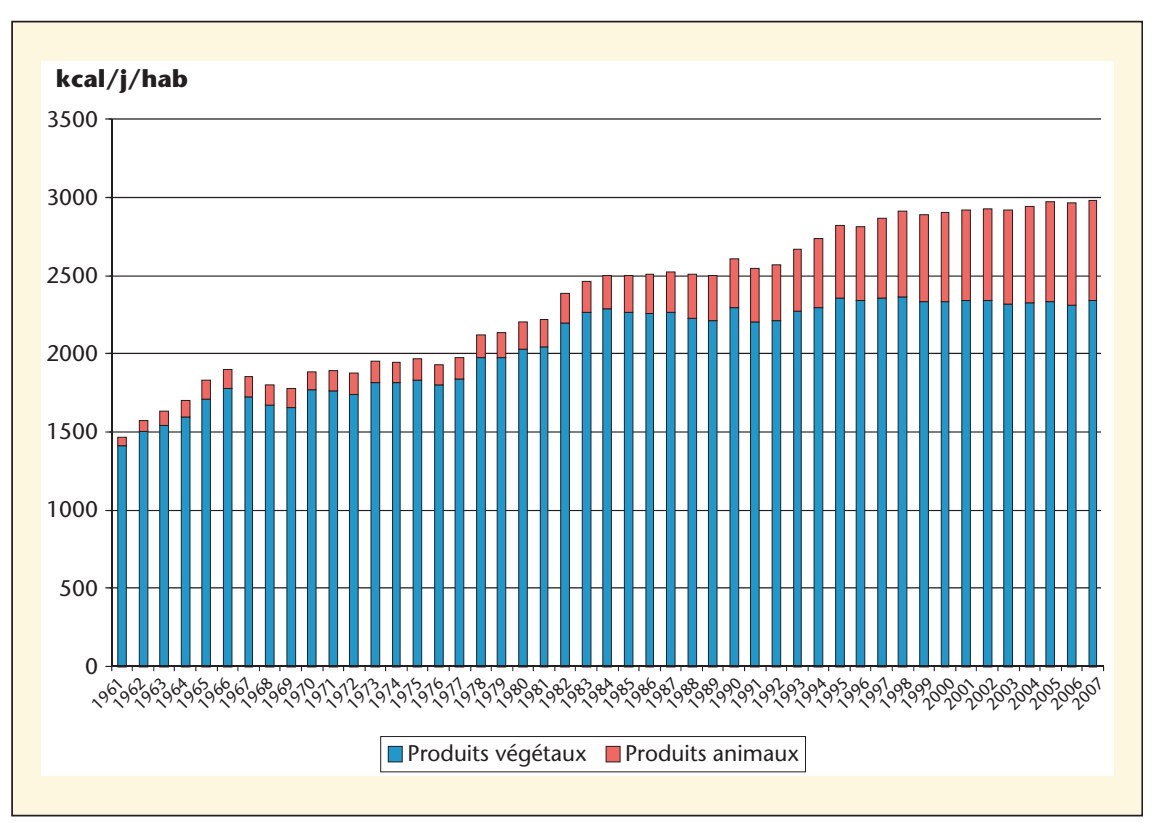

Figure 3. Évolution des disponibilités alimentaires chinoises en kcal/habitant. Source: GEB-Institut de I'Elevage d'après FAOStat blé), de racines amylacées (telles que la pomme de terre ou le manioc) et de légumineuses sèches (pois, haricots...), le reste des produits végétaux étant constitué d'oléagineux, de cultures sucrières, de stimulants (thé, café). Dixsept ans plus tard, au début de la politique de réforme et d'ouverture, les disponibilités alimentaires atteignaient près de $2200 \mathrm{kcal} / \mathrm{j} / \mathrm{habitant}$, mais leur structure n'avait pratiquement pas été modifiée (figure 3 ). Cette progression a été rendue possible par l'augmentation conjointe des disponibilités en produits animaux et en produits végétaux, ces derniers représentant encore plus de $90 \%$ des disponibilités totales. Les denrées animales et d'origine animale ne représentaient pas alors plus de $10 \%$ des apports totaux. Plus des trois quarts de l'alimentation étaient donc constitués de glucides, le quart restant se répartissant entre lipides et protéines (figure 4).

Avec I'ouverture de la Chine au tout début des années 1980, une seconde étape, la transition nutritionnelle, se caractérisant par une modification importante de la structure du régime alimentaire, a pu avoir lieu. Les consommateurs chinois sont passés d'une alimentation uniquement centrée sur le végétal à une alimentation variée où la part de l'animal a pris une place importante (figure 3). Mais cette phase, contrairement au cas français, a débuté avant que ne se termine la saturation calorique : l'évolution de la structure est allée de pair avec l'augmentation des disponibilités totales.

Les disponibilités en produits végétaux ont d'abord continué à progresser mais ont rapidement atteint un plateau au milieu des années 1980. Les céréales sont restées la principale source d'énergie mais leur consommation a diminué depuis en proportion comme en quantité ce qui a laissé la place aux oléagineux et dans une moindre mesure aux fruits. La consommation de racines et tubercules a chuté dans le même temps. La progression des disponibilités qui est intervenue ensuite, au cours des années 1980 et surtout 1990, est entièrement due à la hausse des disponibilités en denrées d'origine animale (y compris d'origine aquatique), qui auraient été une multipliées par 5,5 entre 1978 et 2007. Cet accroissement s'est cependant émoussé au milieu des années 2000. Les denrées animales et d'origine 


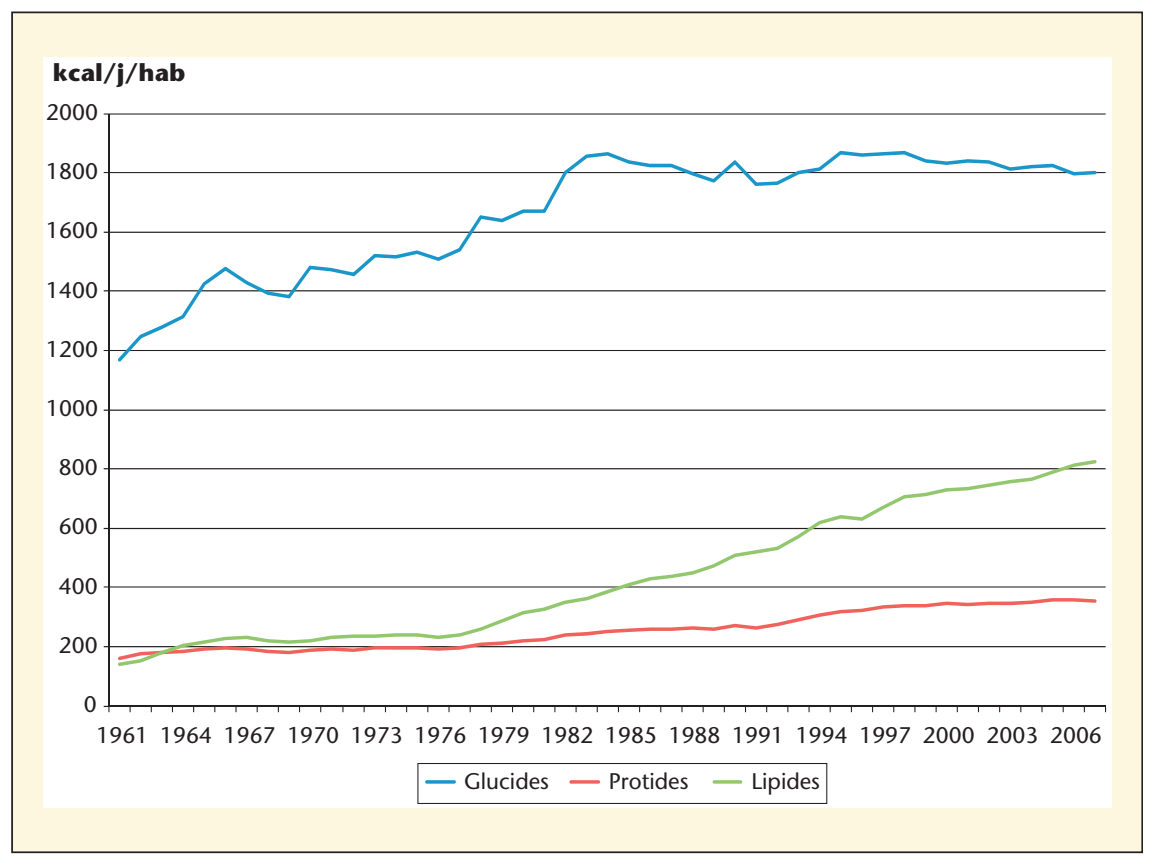

Figure 4. Évolution des disponibilités alimentaires chinoises : répartition par macronutriments (en kcal/habitant).

Source: GEB-Institut de I'Elevage d'après FAOStat

animale représentaient $21 \%$ des disponibilités totales en 2007 . D'un point de vue énergétique, les lipides sont en grande partie responsables de la hausse de disponibilités à partir de 1978 (figure 4), leur consommation ayant été multipliée par 3 au cours des 30 dernières années. Ils constituent aujour$\mathrm{d}^{\prime}$ hui plus du quart des disponibilités alimentaires chinoises, contre plus du tiers en France, et semblent poursuivre leur progression. On constate dans le même temps, une stabilisation voire une baisse des disponibilités en glucides.

En 2007, les disponibilités totales approchent les $3000 \mathrm{kcal} / \mathrm{j} / \mathrm{hab}$. et ont peu progressé depuis la fin des années 90 . La Chine est-elle arrivée à une stabilisation de son niveau calorique global? La transition alimentaire chinoise a été beaucoup plus rapide que dans les pays occidentaux. S'il a fallu plus de 100 ans à la France pour atteindre sa saturation calorique (Combris et Soler, 2011), la Chine semble y être arrivée en moins de 40 ans. La hausse des calories d'origine animale est donc responsable de la modification de la ration alimentaire chinoise. Mais les grands équilibres entre les types de protéines animales n'ont que peu évolué (figure 5). La viande porcine reste la plus consommée désormais non agricole (salaires des ouvriers des entreprises rurales ou des migrants principalement). Selon les données du National Bureau of Statistics, l'écart était de 2,5 en 1980 avant de se réduire à 1,9 en 1985 puis de progresser à nouveau pour atteindre 3,33 en 2009. Depuis, le différentiel a officiellement régressé pour ne plus être que de 3,12 en 2011. Les urbains gagnent donc actuellement trois fois plus que les ruraux.

L'écart réel de revenu est sans doute sous-estimé par les chiffres officiels (Li et Luo, 2010). D'un côté, si le calcul du revenu des ruraux prend en compte I'autoconsommation (sans pour autant connaître les prix utilisés), les revenus des urbains n'incluent pas différentes subventions dont ils bénéficient (médicales, éducation, logement) ainsi que les contributions à la sécurité sociale (cotisations chômage, cotisations assurance-maladie, cotisations retraites...). D'un autre côté, les statistiques ne prennent pas en compte les différences de prix entre les zones urbaines et rurales et il est par conséquent délicat de comparer le pouvoir d'achat réel des consommateurs urbains et ruraux. disponibilités se seraient accrues respectivement de plus de $360 \%$ et de plus de $690 \%$ depuis les années 60 . Les progressions les plus importantes auraient été réalisées par les produits laitiers et la viande bovine (+ $1300 \%$ et $+695 \%)$, des produits traditionnellement peu produits et peu consommés en Chine, sauf dans les zones pastorales du nord et l'ouest du pays. L'ouverture de l'Empire du Milieu aux modes de vie et de consommation étrangers, à partir des années 1980-90 ainsi que les efforts des autorités chinoises pour promouvoir ces produits, ont peu à peu orienté une partie de la consommation. À noter également la forte progression des produits aquatiques avec une multiplication presque par 6 des disponibilités.

\section{Des disparités sociales et géographiques}

Ces moyennes nationales ne permettent pas de rendre compte des différences sociales et géographiques des consommations alimentaires en Chine. Une première rupture apparaît entre urbains et ruraux. L'écart de revenus entre les deux composantes de la population chinoise ne cesse en effet de s'accroître aux dépens des ruraux (figure 6) dont près de la moitié du revenu est
II n'en reste pas moins que l'écart des revenus explique en grande partie les différences de consommations alimentaires entre urbains et ruraux. La part des revenus consacrée à l'alimentation reste, en 2010, plus importante en milieu rural (41\%) qu'en milieu urbain (36\%), à comparer à une moyenne française de $14 \%$. Les achats des ménages ruraux en viandes (porc, bœuf, mouton et volaille) sont estimés par les autorités chinoises à $24 \mathrm{~kg} / \mathrm{an} / \mathrm{hab}$. en moyenne alors que les achats urbains seraient de $34 \mathrm{~kg} / \mathrm{an} /$ hab., soit un écart de $40 \%$. Cependant, ces chiffres ne prennent pas en compte la restauration hors domicile et sous-estiment donc la consommation totale, et surtout de viandes (Waldron et al., 2007). La consommation hors domicile en ville est estimée en moyenne à $20 \%$ de la consommation totale (Gale et Huang, 2007), avec une proportion qui peut aller du simple au double selon les villes (Bai et al., 2012). À l'inverse de la viande, les achats de grains ${ }^{4}$ par les

\footnotetext{
${ }^{4}$ Cette production comprend, selon la terminologie chinoise, les céréales, les légumineuses et les tubercules, ces derniers pour $1 / 5^{\mathrm{e}}$ de leur poids.
} 


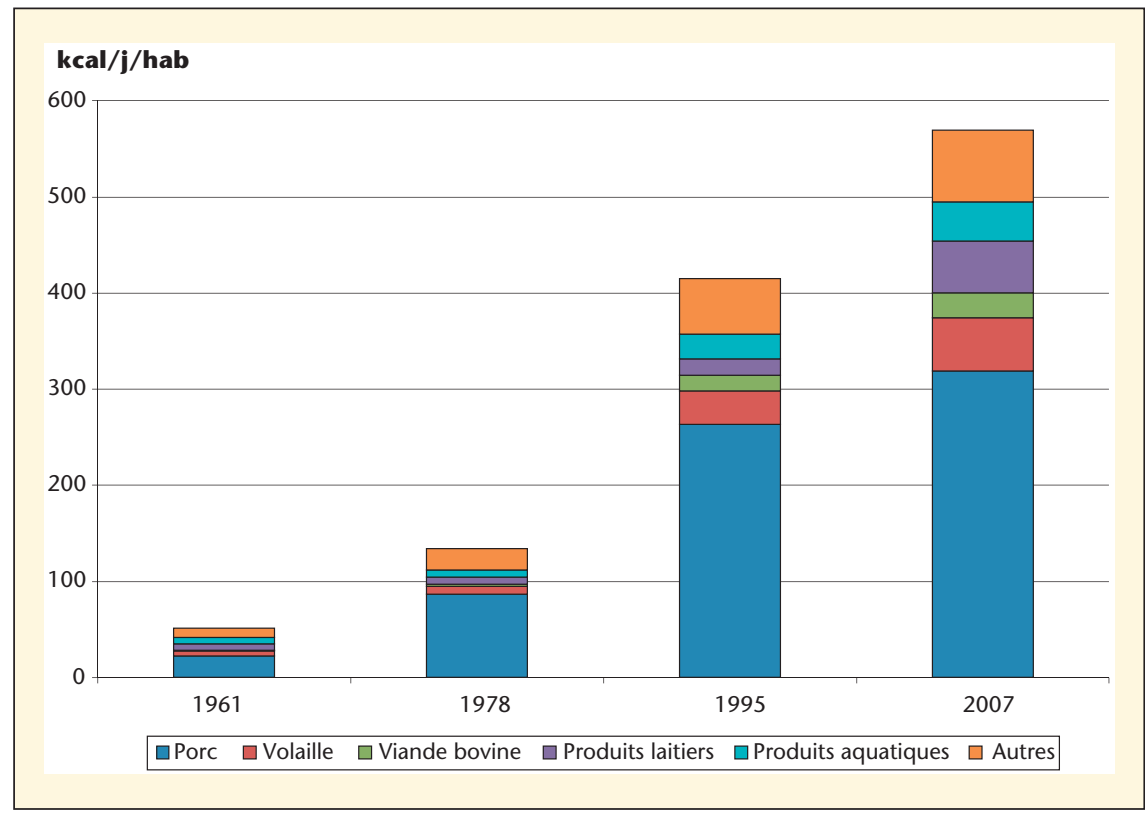

Figure 5. Évolution des disponibilités en produits animaux en Chine (en kcal/habitant). Source : GEB-Institut de I'Elevage d'après FAOStat

urbains seraient de $81 \mathrm{~kg}$ par an tandis que les ruraux consommeraient plus de $180 \mathrm{~kg}$ de grains non transformés.

D'autres facteurs peuvent renforcer ces différences de consommations. Le retard en termes d'infrastructures et de communication entre la production et la distribution alimentaire représentent des freins à la consommation des ruraux dans certaines régions (Yang, 2011). Ainsi, certains aliments, comme la viande bovine, les fruits ou les œufs sont, en moyenne, plus consommés par les plus pauvres des urbains que par les plus riches des ruraux. L'écart est tel que les consommations alimentaires des ruraux atteignent tout juste le niveau de celles des urbains des années 1980.

Le lieu de résidence influe également beaucoup sur les revenus et donc sur les consommations. La croissance chinoise a en effet été longtemps géographique-

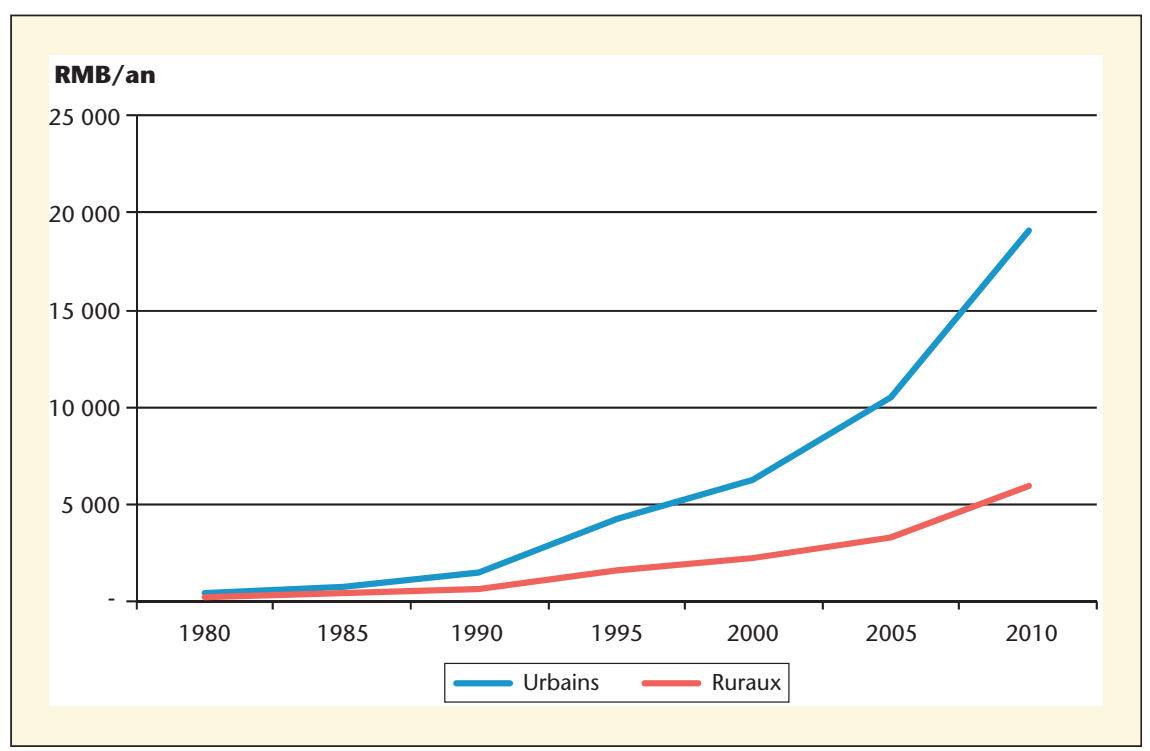

Figure 6. Évolution des revenus ruraux et urbains en Chine (en RMB courant/an). Source: GEB-Institut de I'Elevage d'après Annuaire Statistique Chinois, NBS

ment concentrée, les provinces côtières bénéficiant en priorité de l'ouverture et des investissements. Si le rattrapage de la croissance semble préoccuper les autorités chinoises qui incitent les investissements à se déplacer dans l'ouest du pays, de grandes différences de revenus persistent entre les provinces chinoises. L'écart de revenu moyen entre Pékin, et la province la plus pauvre, le Gansu, était par exemple de $130 \%$ en 2010. Ces contrastes sont également visibles en termes de modes de consommation alimentaire. La part du revenu urbain consacré aux achats alimentaires est de $50 \%$ au Tibet et de seulement $32 \%$ à Pékin (pour une moyenne nationale de $36 \%$ ). En milieu rural, les extrêmes vont de $32 \%$ dans la municipalité de Pékin (qui compte des zones rurales) à $50 \%$ sur l'île de Hainan (pour une moyenne nationale de dépenses alimentaires de $41 \%)$. La part des différents produits alimentaires est également très variable selon les régions. Les consommateurs ruraux du Guangdong achètent $33 \mathrm{~kg}$ de viande (porc, bœuf, mouton et volaille) par an contre seulement $7 \mathrm{~kg}$ dans le Shanxi.

Les revenus n'expliquent pas en totalité les différences de consommation entre les différentes provinces. La géographie de la production alimentaire joue encore un rôle avec, par exemple, la pisciculture et la pêche à proximité des provinces côtières de l'Est ou l'élevage pastoral en Mongolie Intérieure qui facilite la consommation des aliments issus de ces productions. Enfin, il ne faut pas oublier que le fossé entre les consommateurs les plus riches et les plus pauvres se mesure également en termes de santé publique. Si $10 \%$ de la population chinoise restaient encore sous-alimentés en 2008, selon la Banque Mondiale, I'Organisation Mondiale de la Santé évalue à 38,5\% la proportion de Chinois de plus de 15 ans en surpoids. Ce chiffre est en forte hausse depuis 2002 (+ $50 \%)$. Ce "double fardeau" se transformerait même en un "triple fardeau ", si I'on y ajoute les carences en micronutriments qui touchent une partie de la population (Cheng, 2007).

\section{Des facteurs de changement toujours à l'œuvre}

Si la croissance de la production agricole a, dans un premier temps, fourni aux consommateurs des produits variés en plus grande quantité, les évolutions du 
régime alimentaire chinois ont ensuite, directement ou indirectement, été la conséquence de l'ouverture de la Chine et de son insertion dans la mondialisation. L'augmentation des revenus, grâce à la croissance économique, est un des tout premiers facteurs expliquant cette transition alimentaire. Aujourd'hui soucieux de développer la consommation intérieure, le gouvernement souhaite développer le pouvoir d'achat. II incite à des hausses de salaires, accorde des subventions pour les repas des écoliers en milieu rural, tente de mettre en place des systèmes $d$ 'assurance sociale afin de libérer l'épargne des ménages ruraux et a commencé à alléger (pas entièrement. ..) le fardeau des taxes imposées par les autorités locales aux agriculteurs. L'effet hausse du revenu a été accentué par une élasticité-revenu positive pour les produits animaux (Du et al., 2004) et faiblement positive ou négative pour les grains (Zhang et Wang, 2003), fondement de la transition alimentaire. La baisse relative des prix des produits animaux a également contribué à cette évolution (Cheng, 2007).

Un autre aspect réside dans l'urbanisation croissante qui a fait évoluer les préférences alimentaires vers des aliments plus faciles à cuisiner et vers une plus grande part des repas pris hors domicile. Les achats des ménages urbains les plus aisés semblent cependant avoir désormais atteint un plafond quantitatif. En moyenne, leurs achats de viande porcine, de viande ovine, d'œufs et de légumes ont stagné voire baissé entre 2005 et 2010 . Les urbains chinois semblent donc, au moins pour partie, avoir franchi une nouvelle étape où les achats alimentaires se stabilisent en calories mais évoluent en termes de services. L'élasticité prix diminue et le choix des consommateurs semble orienté par d'autres facteurs comme la sécurité du produit, la marque, la nouveauté... (Dong et Fuller, 2010). La restauration hors domicile est très sensible au revenu et fait évoluer le régime alimentaire des urbains les moins aisés vers encore plus de viande (Ma et al., 2006).

La Chine compte de plus en plus de citadins, donc de personnes qui passent du régime alimentaire propre aux zones rurales au régime urbain. En 2011, les autorités chinoises ont déclaré que plus de $50 \%$ de la population du pays vivaient dorénavant en zone urbaine, soit plus de 690 millions d'habitants. Dans le même temps, la population rurale a diminué de 14,5 millions de personnes à 657 millions de ruraux entre 2010 et 2011. Même s'il est parfois difficile de distinguer précisément les Chinois habitant réellement en zone rurale et ou en zone urbaine (Vermander, 2010), la tendance générale ne laisse pas de doute quant à l'ascendant pris par la population urbaine sur la population rurale et qui n'ira qu'en s'accentuant.

La mondialisation a également joué un rôle plus direct dans l'évolution constatée. L'ouverture au commerce international a permis d'importer massivement et $d^{\prime}$ abaisser les prix de produits tels que les huiles qui constituent une part importante des lipides consommés en Chine (Cheng, 2007). Les investissements directs étrangers (IDE) dans la restauration, la transformation et la distribution alimentaire ont aussi contribué à diversifier le régime alimentaire. Modestes dans les années 1980, les IDE dans le secteur agroalimentaire ont fortement augmenté au cours des années 1990. En 1997, les coentreprises représentaient déjà $22 \%$ de la valeur du secteur de la transformation alimentaire (Wei et Cacho, 2001). Le secteur de la restauration n'est pas en reste, à en juger par la percée de McDonald, dont l'Empire du Milieu représente le troisième marché en chiffre d'affaires après les États-Unis et le Japon. Ce phénomène a accentué les différences entre générations anciennes, aux modes alimentaires plus traditionnels, et les jeunes, plus influencés par des modes de consommation occidentaux. Le développement des modes de distribution occidentaux, avec la grande distribution, a également contribué à cette évolution. Le nombre de GMS a été multiplié par 20 entre 1994 et 2002 grâce à la libéralisation du secteur (Hu et al., 2004). Majoritairement situées dans les grandes villes de la côte au début des années 2000, les grandes surfaces ont ensuite peu à peu essaimé dans les villes moyennes ainsi que dans le centre et l'ouest du pays. Par exemple, Auchan et Carrefour ${ }^{5}$ comptent désormais chacun plus de 200 hypermarchés, filiales comprises, répartis dans tout le pays.

\footnotetext{
${ }^{5}$ Source : magazine Linéaires de juillet 2012 : "Les enseignes françaises dans le Monde ".
}

Enfin, la politique alimentaire chinoise a joué un rôle non négligeable. La disparition dès 1985 des coupons de rationnement en ville pour les produits alimentaires non essentiels, en 1993 pour les autres, a libéré la consommation chinoise et permis aux citadins de consommer plus et différemment. La promotion de certains aliments par les autorités chinoises a également beaucoup favorisé l'adoption de nouveaux produits par les consommateurs. La distribution à prix coûtant de produits laitiers dans les écoles en est un exemple.

\section{La capacité de la Chine à s'autosuffire en produits alimentaires en débat}

Les profondes mutations que connaît la Chine depuis la fin des années soixantedix l'ont conduite à s'ouvrir de plus en plus au commerce mondial, une dynamique qui s'est largement amplifiée dès lors que la Chine accédait à l'Organisation Mondiale du Commerce en 2002. Toute une série de conséquences en ont découlé, à commencer par la diminution des barrières douanières, notamment pour les produits agricoles et alimentaires. La question de fond est donc de savoir si cette économie sera ou non davantage tournée vers l'extérieur pour s'approvisionner en produits agricoles et alimentaires dans les décennies à venir.

\section{Une production agricole qui peine à suivre}

Les conséquences de l'évolution de la ration alimentaire chinoise commencent à se faire sentir sur le plan de la sécurité alimentaire du pays. L'objectif d'autosuffisance (95\% des grains, soja mis à part) affiché par les autorités chinoises est chaque année plus difficile à atteindre car le volume de productions agricoles à fournir pour satisfaire la demande croît très vite.

Dans un premier temps, la décollectivisation à la fin des années 1970 a libéré le potentiel agricole longtemps contraint par l'organisation rigide mise en place dans les années 1950. L'agriculture a été le premier secteur à sortir du cadre centralisé et a ainsi précédé l'industrie dans sa métamorphose. Les communes populaires ont été démantelées et les familles rurales ont gagné en liberté de production grâce au "système de 
responsabilité des ménages " et à la décentralisation des décisions de production qui en a découlé. En parallèle, les autorités chinoises ont procédé à une libéralisation graduelle du commerce des produits agricoles. Les produits animaux ont été parmi les premiers, en 1984, à pouvoir être vendus librement à l'intérieur du pays, permettant la création de marchés agricoles et facilitant la circulation des denrées à travers le pays.

Dans le même temps, les augmentations successives des prix agricoles ont largement contribué à la progression spectaculaire des productions céréalières, malgré des quotas de livraison obligatoires restés en place pendant plusieurs décennies et qui ont pris fin en 2004. La même année, dans le but de stimuler à nouveau la production céréalière et soutenir les revenus agricoles, la mise en place de subventions a commencé. Au final, la production de grains a cru plus rapidement que la population (figure 7). La production aurait été multipliée par 1,7 entre 1978 et 2010 tandis que la croissance démographique n'était que de 1,4. La production de grains par habitant serait ainsi passée de $318 \mathrm{~kg}$ en 1978 à $460 \mathrm{~kg}$ en 2010. Dans le même temps, la production de fruits aurait, elle, été multipliée par plus de 30. Ces mesures de politiques agricoles ont permis une forte progression des différentes productions, la Chine cultivant son autosuffisance pendant les 25 années qui ont suivi le début des réformes. Elle a essentiellement pourvu à ses besoins alimentaires, n'important que du blé en quantités relativement importantes dans les années 80 et 90 .

Mais, l'évolution des modes de production agricoles, avec le recul de l'élevage de porc et de volaille de basse-cour et le développement des exploitations de grande taille le plus souvent hors-sol, augmente la part des céréales destinée à I'alimentation animale et renforce la pression sur les terres agricoles. Or, la production agricole est confrontée à de nombreux défis. Les terres cultivables se réduisent face à l'urbanisation, aux réquisitions de terres par les autorités locales et aux infrastructures nécessaires au développement fulgurant du parc automobile chinois. La Chine aurait perdu, 8,2 millions d'hectares de terres depuis 1997 sur une surface totale de 120 millions d'hectares en 2010 (De Schutter, 2010). Une partie importante des terres agricoles restantes est

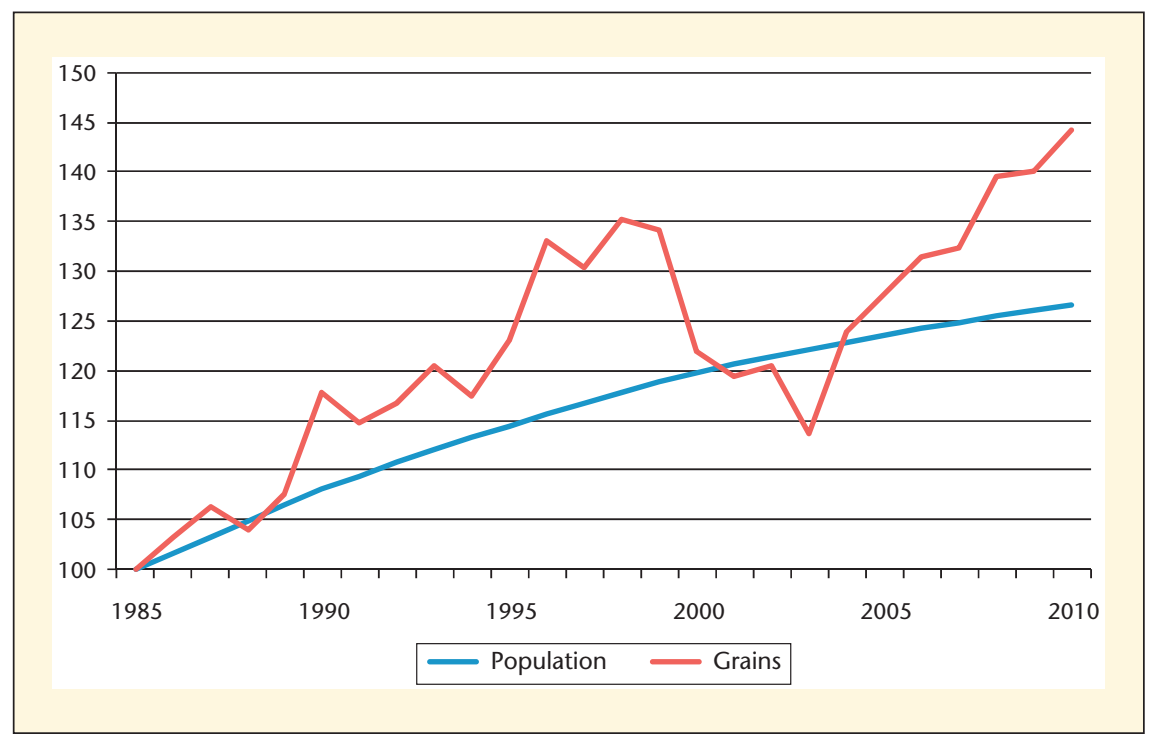

Figure 7. Évolutions comparées de la population chinoise et de la production de grains (1995 = base 100).

Source: GEB-Institut de I'Elevage d'après Annuaire Statistique Chinois, NBS dégradée par l'usage excessif d'engrais ou polluées (métaux lourds...). Les réserves d'eau s'amenuisent rapidement, notamment dans le nord du pays, menaçant l'irrigation nécessaire sur près de $50 \%$ des surfaces cultivées. Face à ces défis, le déficit agricole et agroalimentaire chinois se creuse et la période de relative autarcie agricole semble révolue.

\section{Un déficit commercial agroalimentaire croissant}

L'économie chinoise se distingue par un excédent commercial croissant entre
2002 et 2007. II a avoisiné $7 \%$ du PIB, illustrant à quel point la croissance était jusque-là tirée par les exportations vers le reste du monde. L'excédent commercial est retombé à $4 \%$ du PIB en 2010 (solde à 452 milliards de dollars). Pour l'essentiel, durant cette période, la structure des exportations était dominée par les produits manufacturés, dont la Chine est désormais le premier exportateur mondial (Lemoine et Ünal, 2012). En revanche, le poids des produits agricoles et alimentaires dans les exportations totales chinoises s'est effondré, passant de quelque $23 \%$ en 1980 à $12,7 \%$ en

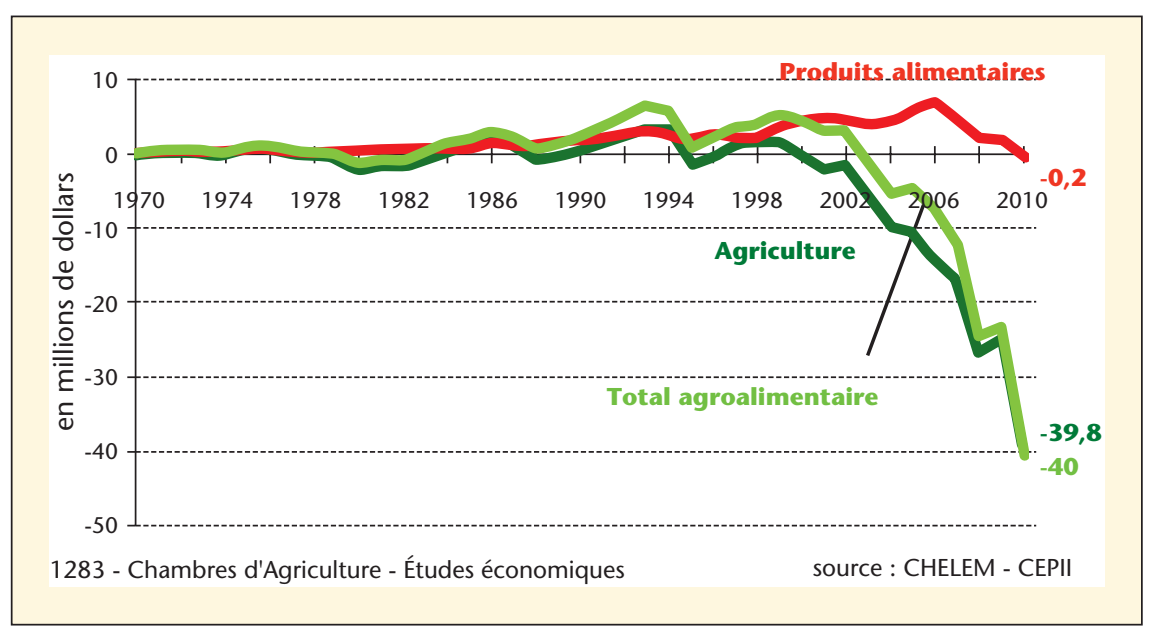

Figure 8. Solde agroalimentaire de la Chine vis-à-vis du monde (en milliards de dollars). 
1990 puis à peine $2,5 \%$ en 2010 . Le poids relatif des importations agricoles et alimentaires, moins élevé que durant les années quatre-vingt, demeure important $(7 \%$ du total des importations en 2010). II en résulte une dégradation régulière du solde commercial agroalimentaire chinois depuis le milieu des années 2000 (figure 8). La dégradation est d'autant plus significative lorsque l'on se penche sur l'indicateur de taux de couverture. La dépendance de la Chine est nette sur les produits agricoles depuis le début des années 2000 , le taux de couverture étant devenu très faible en 2010, seulement 49,9\% (figure 9) . Quels sont les postes de la balance commerciale agroalimentaire chinoise qui ont dégradé à ce point le solde ?

La consommation alimentaire chinoise se porte désormais sur des produits carnés, y compris les produits laitiers, et sur les vins et alcools. Si les besoins en produits carnés peuvent être satisfaits par la production domestique, que l'on pense par exemple à la viande porcine, la Chine importe massivement des végétaux pour nourrir le bétail. C'est ainsi que la Chine s'est retrouvée parmi les plus gros importateurs de soja, en provenance notamment du Brésil et d'Argentine (figure 10). Les graines oléagineuses, les huiles et graisses végétales représentaient en 2010 plus de $45 \%$ des importations chinoises de produits agroalimentaires. En graines de soja, la Chine capte près de $58 \%$ des importations mondiales, sa part étant plus modeste dans les importations mondiales d'huile de palme (17\%), et d'huile de soja $(17 \%)$. L'ouverture commerciale de la Chine est par conséquent manifeste pour des productions comme le soja, mais aussi le coton, le caoutchouc et la pâte à papier.

En produits laitiers, au regard de la croissance de la demande mais aussi en raison de la contamination récente du lait chinois par la mélamine, les importations se sont accrues de quelque $4000 \%$ entre 1990 et 2010 . Ce chiffre, pour impressionnant qu'il soit, doit être nuancé, dans la mesure où les importations de produits laitiers ne représentaient en 2010 qu'à peine $3 \%$ des importations totales agroalimentai-

${ }^{6} \mathrm{TC}=[\mathrm{X} / \mathrm{M}]^{\star} 100$. Un TC supérieur à $100 \%$ indique que l'économie est bien positionnée sur les marchés mondiaux, et moins ou peu spécialisée si le TC est inférieur à $100 \%$.

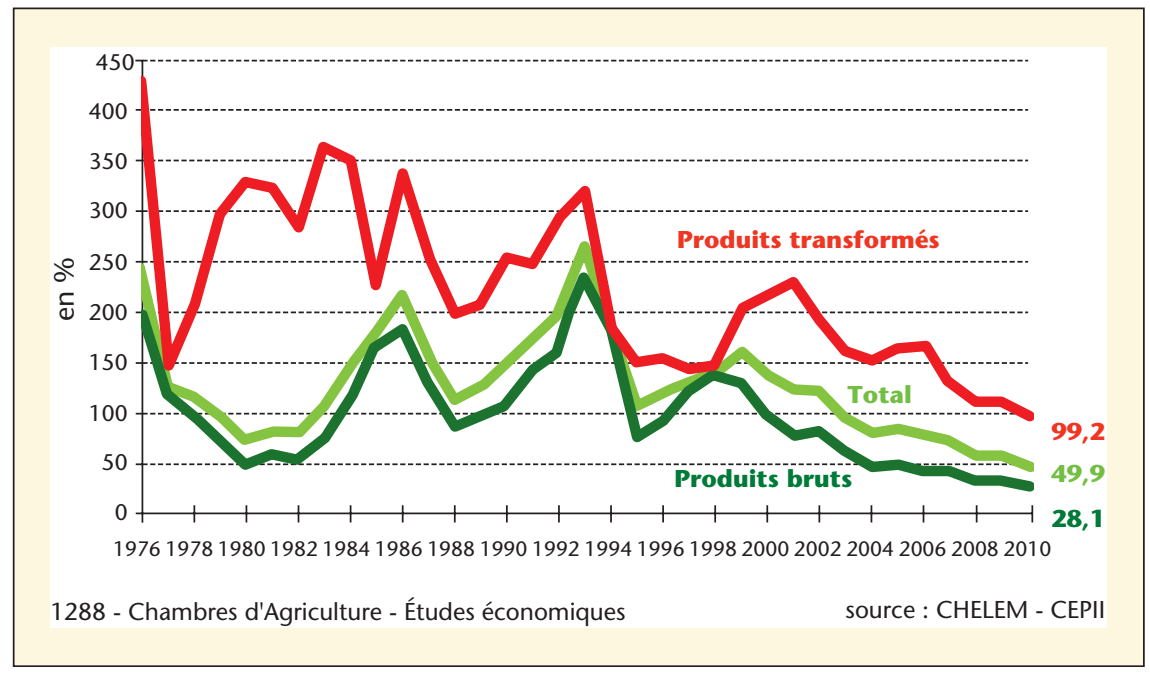

Figure 9. Taux de couverture de la Chine dans le secteur agroalimentaire (en \%).

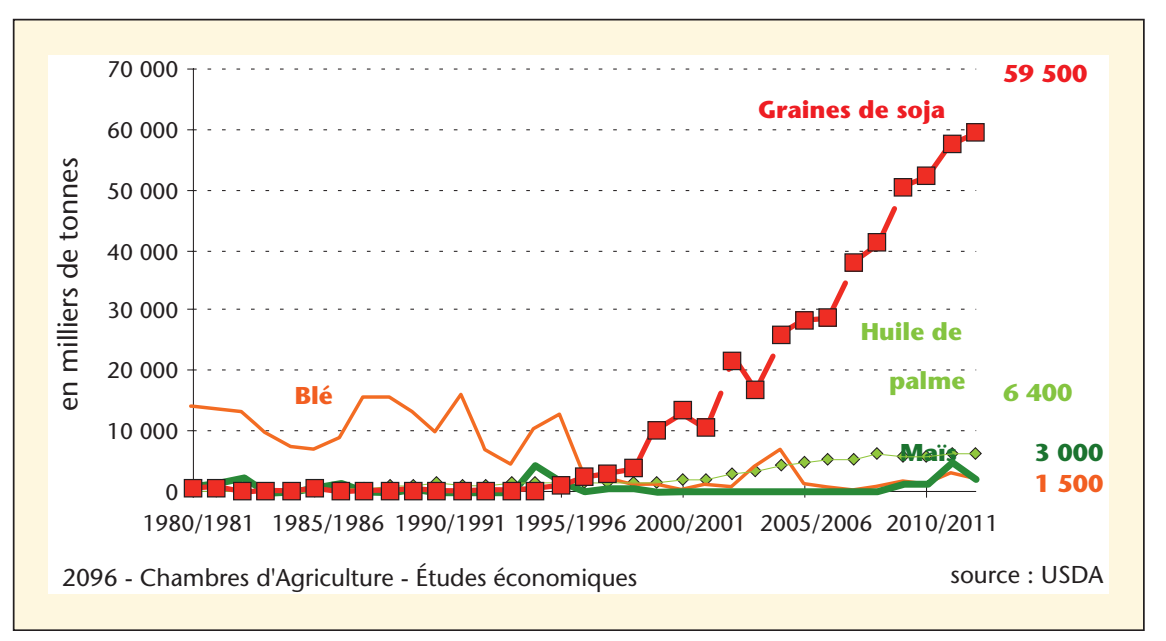

Figure 10. Importations chinoises de produits agricoles (en milliers de tonnes).

res chinoises. La croissance des importations chinoises de produits laitiers entre 2012 et 2011 a été de $81 \%$. Les principaux fournisseurs de la Chine en lait et produits laitiers étaient en 2010 : I'Australie/Nouvelle-Zélande (70\% des exportations mondiales de produits laitiers vers la Chine), suivies des États-Unis $(10,3 \%)$, puis la France avec seulement $4,3 \%$. D'un autre côté, la NouvelleZélande en particulier est devenue très dépendante de ses exportations vers la Chine $(20 \%$ du chiffre d'affaires total de la filière $)^{8}$.

\footnotetext{
${ }^{7}$ Source : CEPII, base de données CHELEM.

${ }^{8}$ Source GEB-Institut de I'Elevage.
}

Dans la phase actuelle de crise, de nombreux économistes estiment que la Chine ne peut fonder durablement sa stratégie de croissance économique sur l'exportation. Elle doit faire converger son essor industriel et l'expansion de son marché intérieur, à l'image de ce que les États-Unis avaient réalisé dès la fin du XIX ${ }^{e}$ siècle (Aglietta, Lemoine, 2010) (Mistral, 2011). Dans le cas du secteur agroalimentaire, il convient de mesurer les conséquences de ce redéploiement de la stratégie chinoise de croissance. Si les productions agricoles ne croissent pas au même rythme que celui de la demande des ménages en biens alimentaires, il est à prévoir que le déficit commercial agroalimentaire 
continue de se creuser dans les années à venir, les exportations de fruits préparés, de jus de pomme, de conserves de légumes, de thé. .. n'étant pas en mesure d'équilibrer la balance commerciale. On comprend mieux alors la politique chinoise de sécurisation des approvisionnements alimentaires, laquelle passe notamment par des acquisitions de terres agricoles en Afrique, ou par des projets d'accords de libre-échange avec la zone MERCOSUR, laquelle est désormais le premier fournisseur de produits agricoles et alimentaires de la Chine.

\section{Conclusion}

La transition alimentaire, qui a débuté il y a 30 ans, n'est pas encore terminée pour une part importante de la population chinoise. Les ruraux et les urbains à faibles revenus disposent d'une marge de progression relativement importante qui devrait être comblée dans les décennies à venir, pour peu que la croissance économique et la redistribution des revenus le permettent. Or, la Chine vient de devenir le deuxième importateur mondial de produits agricoles et agroalimentaires, derrière I'UE. Si le rôle de la Chine dans les émeutes de la faim en 2008 semble avoir été très limité, l'évolution prévisible de sa demande mène à s'interroger sur son rôle futur sur les marchés alimentaires internationaux des produits à faible intensité de main-d'œuvre.

Remerciements. Les auteurs tiennent à remercier Philippe Chotteau, responsable du département économie de I'Institut de I'Elevage pour sa relecture attentive et constructive. Les auteurs restent responsables de toute erreur ou omission qui subsisterait.

\section{Conflits d'intérêts : aucun}

\section{RÉFÉRENCES}

Aglietta M, Lemoine F. La nouvelle frontière de l'économie chinoise. In : CEPII (éd.),
L'économie mondiale 2011. Editions La Découverte, coll. "Repères " : 32-49, 2010.

Apoteker T. La Chine: accélération des transformations et nouveaux défis. Revue d'économie financière 2009 ; (95), deuxième trimestre : 207-28.

Aubert C. Sécurité alimentaire et tendances de la consommation en Chine. Perspectives Chinoises $2008 ; 2$ : 5-25.

Bai J, Seale J, Lohmar B, Wahl T. Meat Demand In Urban Chinese Households. GAIN Report, USDA, 2012.

Brown L. Who Will Feed China? World Watch Institute. W.W. Norton, 1995.

Combris P, Soler LG. Consommations alimentaires: tendances de long terme et questions sur leur durabilité. Innovations agronomiques 2011 ; (13) : 149-60.

Cheng F. The Nutrition Transition and Obesity in China. Case Study n 3-9 of the program: "Food policy for developing countries: the role of government in the global food system". Cornell University, 2007.

De Schutter O. Mission to the People's Republic of China from 15 to 23 December 2010. Preliminary observations and conclusions, United Nations, 2010.

Dong F, Fuller F. Dietary Structural change in China's cities: empirical fact or urban legend? Canadian Journal of Agricultural Economics 2010 ; 58 : 73-91.

Du S, Mroz TA, Zhai F, Popkin BM. Rapid income growth adversely affects diet quality in China-Particularly for the Poor! Social Science and Medecine 2004 ; 59 : 1505-15.

Fu J, Wang Z, Wang B. Transitions of food groups and nutrients in the northeast of China: A 3-year-interval's follow-up study. The Internet Journal of Nutrition and Wellness 2006 ; 2 (2).

Gale F, Huang K. Demand for food quantity and quality in China. Economic Research Service 2007 ; (32), USDA.

He Y, Simon JC. La dynamique agricole chinoise face au commerce mondial: un nouveau Big Bang?" Revue Tiers-Monde $2005 ; 46: 517-37$.

Hu D, Reardon T, Rozelle S, Timmer P, Wang $\mathrm{H}$. The emergence of supermarkets with Chinese characteristics: Challenges and the opportunities for China's agricultural development. Development Policy Review 2004 ; 22 : 557-86.
Lemoine F. La Chine et I'Inde : les plus solides des BRIC. Revue d'économie financiere 2009 ; (95), deuxième trimestre : 229-41.

Lemoine $F$, Ünal D. Rééquilibrage du commerce extérieur chinois. La Lettre du CEPII 2012 ; (320) : 1-4.

Li S, Luo C. Re-estimating the income gap between urban and rural households in China. Procedia Social and Behavorial Sciences 2010 : 7151-63.

Ma H, Huang J, Rozelle S. Reassessing China's livestock statistics: an analysis of discrepancies and the creation of new data series. Economic Development and Cultural Change $2004 ; 52: 445-73$.

Ma H, Huang J, Fuller F, Rozelle S. Getting rich and eating out: consumption of food away from home in urban China. Canadian Journal of Agricultural Economics 2006 ; (54) : 101-9.

Mistral J. La réorientation de la croissance chinoise. Sa logique, ses enjeux et ses conséquences. Note de I'IFRI. IFRI, Centre d'études économiques, 2011 : 1-38.

Sabban F. Transition nutritionnelle et histoire de la consommation laitière en Chine. Cholédoc $2010 ; 120$.

Vermander B. Chinois des villes et Chinois des champs.... Chine Plus 2010 ; (13) : 22.

Waldron SA, Brown CG, Zhang CG. Update on developments in the chinese cattle and beef industry of relevance to the australian industry. Meat and Livestock Australia Report 2007, 260 pages.

Wei A, Cacho J. Competition among foreign and chinese agro-food enterprises in the process of globalization. International Food and Agribusiness Management Review 2001 ; 2 : 437-51.

Yang Y. Study on characteristics of consumption demand and its constraints of rural areas in China. Asian Agricultural Research 2011; 3: 87-89, 93.

Zhai F, Wang H, Du S, He Y, Wang Z, GE K, Popkin BM. Prospective Study on Nutrition Transition in China. Nutrition Reviews 2009; 67 (Suppl. 1) : S56-S61.

Zhang W, Wang Q. Changes in China's food consumption and implication for trade. Paper prepared for presentation at the American Agricultural Economics Association Annual Meeting, Montreal Canada, July 2730, 2003. 